\title{
EL PAPEL DE LA EDUCACIÓN EN CREACIÓN DE EMPRESAS EN EL CONTEXTO UNIVERSITARIO A PARTIR DE LOS ESTUDIOS REGISTRADOS EN SCOPUS
}

\author{
Role of entrepreneurship education in the university context from studies registered in Scopus
}

Juan Pablo Mejía Ordoñez

Ingeniero Administrador, Universidad Nacional de Colombia, jpmejiao@unal.edu.co

Cristian Manuel Arias Marín

Estudiante Gestión Turística, Colegio Mayor de Antioquia, cristariasm@gmail.com

\section{Laura Cristina Echeverri Sánchez}

Estudiante de la Especialización en Analítica e Ingeniera Administradora, Institución Universitaria Escolme, cies2@escolme.edu.co

\begin{abstract}
Cómo citar / How to cite
Mejía-Ordoñez, J. P, Arias-Marín, C. M. y Echeverri-Sánchez, Laura Cristina (2017). El papel de la educación en creación de empresas en el contexto universitario a partir de los estudios registrados en Scopus. Revista CEA, 3(5), 69-87.
\end{abstract}

Recibido: 30 de agosto de 2016

Aceptado: 30 de octubre de 2016

\begin{abstract}
Resumen
Las comunidades universitarias han realizado sinnúmero de investigaciones de los fenómenos que enmarcan educación emprendedora, sin lograr aun un consenso que permita trazar líneas de ruta en investigaciones futuras. Es por lo anterior que este artículo de revisión tiene por objetivo analizar las tendencias investigativas sobre el papel de la educación emprendedora en un periodo de 15 años (1999 al 2014), a través de un análisis bibliométrico de la producción investigativa registrada en Scopus, así, presentar una conceptualización de la terminología más relevante en el tema de emprendimiento $y$, finalmente, discutir los hallazgos de los estudios hasta ahora adelantados, de tal manera que sea posible visualizar líneas investigativas a futuro en esta temática.
\end{abstract}

Palabras clave: bibliometría, educación emprendedora, instituciones de educación superior.

\begin{abstract}
The university communities have carried out countless investigations of the phenomena that frame entrepreneurial education; without achieving even a consensus that allows to draw lines of route in future investigations. It is for the above that this review article has as purpose to analyze the investigative tendencies on the role of the entrepreneurial education in a period of 15 years (1999 to 2014) through a bibliometric analysis of the research production registered in Scopus, in order to present a conceptualization of the most relevant terminology in the topic of entrepreneurship and finally discuss the findings of studies, so far advanced. In such a way that it is possible to visualize future research lines in this area.
\end{abstract}

Keywords: bibliometrics, entrepreneurship education, institutions of higher education. 


\section{INTRODUCCIÓN}

El emprendimiento ha sido reconocido como una de las fuerzas detrás del desarrollo económico (Naudé, 2013; Horvath, 2016) y social (Wompner, 2008), las cuales son impulsadas desde la innovación, generando nuevas áreas de negocio que han traído al mercado productos completamente nuevos (Piñuel, 2014). En este sentido, se ha señalado que el emprendimiento desempeña un importante rol en la contribución del crecimiento económico de las regiones (Panigrahi y Joshi, 2015; Arshad, Farooq, Sultana y Farooq, 2016), y ha sido aún más trascendental para los países en desarrollo como medio para atenuar diversos problemas económicos (Siddiqui y Jan, 2017), tales como el desempleo (Panigrahi y Joshi, 2015).

Se ha visto que el crecimiento del emprendimiento entre personas menores de 30 años ha generado cambios en la dinámica global de desarrollo y creación de empresas y, a su vez, la forma como se percibe la importancia de la iniciativa en la sociedad (Kuratko, Ireland, Covin y Hornsby, 2005). En este contexto, se ha resaltado la importancia de estimular a los jóvenes hacia la actividad emprendedora, pues son ellos los futuros empresarios y como tal está ligado el crecimiento futuro de las regiones (Kyrgidou, Mylonas y Petridou, 2013).

Dado a lo anterior, la educación empresarial ha recibido gran atención (Matsheke y Dhurup, 2017) debido a su rol en el desarrollo de los futuros empresarios (Velasco, 2016), al servir como instrumento para motivar a las personas hacia la acción emprendedora. En la literatura se han abordado diversas investigaciones en torno al papel de la educación empresarial sobre la intención empresarial (Entrialgo e Iglesias, 2016), la cual es considerada como un requisito previo para iniciar un negocio (Soria-Barreto, Zuniga-Jara y Ruiz-Campo, 2016).

Este tipo de educación ha sido parte de la educación de negocios desde 1970 (Kuratko, 2003), y sigue siendo un punto importante en las políticas educativas universitarias a nivel mundial (Mahieu, 2006). Desde la década de 1980, también se ha promovido el emprendimiento en la educación primaria, secundaria y en pregrado, ya que se considera como una respuesta a las necesidades importantes en la sociedad y la educación (Riese, 2011), por lo que resulta fundamental la integración de la educación emprendedora en todo el sistema educativo, con el fin de que se convierta en un modo de vida (Sun, 2017). Esto ha generado una explosión en términos de iniciativas públicas y privadas al promover la actividad emprendedora, impulsado con el objetivo de acelerar la innovación, el desarrollo tecnológico y la creación de empleo (Rasmussen y Sørheim, 2006).

El concepto de universidad emprendedora se introdujo a principios de la década de 1980 invitando a las instituciones de educación superior a contribuir de forma más abierta al crecimiento económico de las regiones (Bienkowska y Klofsten, 2012). Los estudios realizados por autores como Saxenian (1994) y Etzkowitz y Klofsten (2005) muestran que la presencia de una universidad en una región particular, durante un largo período de tiempo, puede tener efectos positivos en el desarrollo regional.

La universidad emprendedora es definida como una institución de educación superior con significativas particularidades, la cual se encuentra vinculada con el entorno y responde de manera proactiva a las necesidades del mercado, sin dejar de lado sus valores académicos ni su misión tradicional como entidad educativa que trabaja con el conocimiento (Chamorro, Ceballos y Hernández, 2012). La universidad emprendedora, más que un bien económico objeto de intercambio, recurre al conocimiento como un potencial al servicio de los objetivos de un entorno socioeconómico, esto es, un recurso que, adecuadamente gestionado, le permite desempeñar un papel más activo en su contexto social (Clark, 2004). 
Dentro de los principales beneficios que se han encontrado sobre la educación en emprendimiento, en el contexto universitario, se encuentra la comprensión de la viabilidad de una nueva idea de empresa, el conocimiento, las habilidades empresariales, una apreciación de lo que es ser un empresario y el aumento de la confianza (Kirkwood, Dwyer y Gray, 2014).

Las organizaciones gubernamentales están recurriendo cada vez más a programas de formación empresarial, con el objetivo de ayudar a las personas a desarrollar competencias necesarias para la generación del autoempleo: creación de su propio negocio (Wiger, Chapman, Baxter y DeJaeghere, 2015). En este contexto, el gobierno debe promover un sistema de educación empresarial fuerte y uestablecer buenos centros de estudio con personal docente bien calificado para educar y capacitar a los posibles emprendedores y poner a su disposición fondos al final del programa de formación empresarial» (Panigrahi y Joshi, 2015).

De esta manera, se ha planteado la educación empresarial como medio para «motivar a los jóvenes a iniciar su propio negocio como una alternativa viable a la integración exitosa de los graduados universitarios en el mercado laboral» (Herman y Stefanescu, 2017, p. 312). Mediante este tipo de educación en las universidades, se fomenta la intención y actitud empresarial de los estudiantes, al tiempo que estos logran entender mucho más el proceso emprendedor (Monzurul, Rafiul y Rana, 2017). No obstante, aunque la investigación académica ha demostrado que la educación empresarial estimula la intención empresarial, este hecho no ocurre uniformemente en todos los contextos (Maresch, Harms, Kailer y Wimmer-Wurm, 2016). Además, este tipo de educación no garantiza la vinculación de los estudiantes con el mundo empresarial, dado que gran parte de los graduados se inclinan directamente por ser empleados en lugar de crear su propia empresa, lo que es originado principalmente por la incertidumbre y riesgos que están asociados a emprender (Abdullah y Abidin, 2014).
El reciente crecimiento y desarrollo en los planes de estudio y programas dedicados a la iniciativa empresarial y la creación de empresas han sido notables, inclusive algunas universidades han optado por incluir la educación empresarial como una materia obligatoria (Hamzah, Yahya, Sarip y Mohd Adnan, 2016) con el fin de reforzar habilidades y capacidades empresariales de los estudiantes (Guerrero, Urbano, Cunningham y Organ, 2014). Sin embargo, en medio de esta expansión de los cursos, el reto de la enseñanza del espíritu empresarial sigue siendo el incremento de la eficacia de los cursos que se imparten (Kuratko, 2003). En este aspecto, se ha señalado que la enseñanza práctica en lugar de los enfoques tradicionales basados en el aula, favorecen la educación empresarial (Nieuwenhuizen, Groenewald, Davids, Rensburg y Schachtebeck, 2016), por lo que muchas iniciativas de educación sobre emprendimiento se están volviendo cada más orientadas a la acción, es decir, fomentando el aprendizaje mediante práctica (Rasmussen y Sørheim, 2006).

Al respecto, se observa que el impacto de la educación empresarial ha sido reconocido como un factor crucial que permite a los jóvenes comprender y fomentar una actitud emprendedora. Esta influencia ha llevado a la necesidad de identificar cómo desarrollar y nutrir los empresarios potenciales, incluso cuando aún son estudiantes en la escuela (Wang y Wong, 2004). Frente a esto, la educación empresarial proporciona un medio a través del cual la cultura institucional puede cambiar, permitiendo un entorno más apropiado en el que puede generarse emprendimiento desde un contexto académico (Jacob, Lundqvist y Hellsmark, 2003). La relación es de doble vía, y, por lo tanto, se requiere más investigación empírica que explore estos aspectos (Pittaway y Cope, 2007).

Por otra parte, el interés por el comportamiento de la producción científica, entendido como indicador de calidad y criterio para comparar el desarrollo y avance de una disciplina o área del conocimiento (Agudelo, Bretón y Buela-Casal, 
2003) ha dado lugar al fortalecimiento de los estudios bibliométricos, los cuales partiendo desde una perspectiva principalmente cuantitativa, a través del uso prudente y un proceso analítico e interpretativo riguroso pueden constituir un importante criterio para determinar las tendencias fundamentales de la dinámica investigativa de las temáticas de estudio (Buela-Casal, Carretero-Dios y De los Santos-Roig, 2002).

Los estudios bibliométricos representan un importante elemento dentro de la literatura científica, dado que ofrecen un claro punto de partida que orienta tanto a lectores como investigadores, a la luz de diferentes asuntos académicos, como las temáticas que mayor acogida tienen en determinada revista o el índice de colaboración que hay en los artículos que se publican, o las citas que reciben determinados artículos (Agudelo et al., 2003). Sin embargo, no puede desconocerse que los datos cuantitativos utilizados en la actualidad para el desarrollo de los análisis bibliométricos no representan el único indicador de calidad de la productividad de una línea de investigación científica, y que, como consecuencia de ello, los análisis bibliométricos pueden resultar significativamente controversiales (López-Cózar, Torres-Salinas, Jiménez-Contreras y Ruiz-Pérez, 2006).

Sin embargo, a pesar de la reconocida importancia de los estudios bibliométricos para la comprensión y la orientación de las diferentes líneas de investigación científica, en la actualidad no se cuenta con muchos estudios en el campo de la formación emprendedora (Virgen-Ortiz, Oliveros y Betancourt-Guerrero, 2014) que ofrezcan valores agregados, como la clasificación tanto temática como metodológica de las investigaciones realizadas, dar una visión diacrónica del desarrollo de los estudios, caracterizar las investigaciones frente a la educación emprendedora conforme a la normativa de la investigación científica y/o evidenciar la productividad de las investigaciones realizadas frente a la educación emprendedora (Cano y Sánchez, 1998).
Es por ello que se plantea como el objetivo de este estudio, realizar un análisis bibliométrico de las tendencias que se han venido presentando en los últimos años (1999 a 2014) en cuanto a investigaciones del papel de la educación emprendedora en ambientes universitarios, con el fin de conocer la productividad de esta línea de investigación y visualizar los nuevos retos y perspectivas que se están generando alrededor de esta importante temática.

\section{METOdOLOGÍA}

El presente estudio examina las relaciones existentes entre las investigaciones de la educación emprendedora en los contextos universitarios, a través de la perspectiva científica que ofrecen los análisis bibliométricos de las investigaciones publicadas a través de Scopus, durante un periodo de 15 años (Cano y Sánchez, 1998).

Por medio de una revisión cienciométrica que se centra en el análisis de la literatura disponible en la base de datos Scopus, en marcada en el periodo de tiempo 1999 a 2014, se trata de crear una visión general y sistemática de cómo se ha venido irrumpiendo en el contexto de la educación emprendedora en los contextos universitarios, a partir de un análisis bibliométrico que recoge un conjunto de métodos matemáticos y estadísticos, usados para analizar y medir la cantidad y calidad de artículos y otras formas de publicaciones científicas (Cadavid, Awad y Franco, 2012).

Se ha escogido la base de Scopus, ya que es la mayor base de datos con alrededor de 20500 publicaciones procedentes de más de 5000 editoriales internacionales, por lo que recopila publicaciones disponibles en otras bases. Adicionalmente, representa aproximadamente un $80 \%$ de las publicaciones internacionales revisadas por especialistas, permitiendo asegurar un contenido representativo y actualizado (Universidad de Granada, 2017). Adicionalmente, Scopus ofrece acceso a 
diferentes bases de datos interdisciplinarias, proporciona herramientas para gestionar la información y cumple otros criterios, como la cantidad de citaciones y la accesibilidad que la hacen ser una de las más usada en este tipo de análisis en la literatura (Boeris, 2011). Por lo tanto, se ha seleccionado Scopus como principal fuente de información, debido a que concede mecanismos para gestionar la información, entrega resultados precisos y coherentes con la matriz de búsqueda y ofrece acceso a diferentes áreas proporcionando herramientas para gestionar la información (Hall, 2011).

Desde esta perspectiva, al utilizarla es posible identificar los principales cambios o las características de un tema específico; explorar, organizar y analizar gran cantidad de datos históricos; encontrar patrones -no evidentesútiles para el avance de las investigaciones y el desarrollo científico (Bouyssou y Marchant, 2011; Cadavid et al., 2012). Para esto se construyó una ecuación de búsqueda que combina los términos entrepreneur*, education", university, learning* e intention en un periodo de 15 años (1999 al 2014).

El uso de las ecuaciones de búsqueda permite la obtención de información útil, de calidad y precisa; particularmente en el contexto de las bases de datos, las ecuaciones de búsqueda radican en la correcta integración de conceptos clave y operadores que permiten expresar una necesidad de información, un proceso búsqueda correctamente direccionado (Sanz-Valero et al., 2008).

Para el presente estudio, se ha definido que los descriptores más pertinentes para llevar a cabo la búsqueda serán las palabras: entrepreneur*, education", university, learning* e intention*, entre los cuales se define como el descriptor principal la palabra "Entrepreneur" y que a su vez será analizada a través de las combinaciones fundamentales con las palabras "education", "student" e "intention" de lo cual se obtienen los siguientes grupos de descriptores relacionados con de educación emprendedora:
Entrepreneur (Emprendedor). Término acuñado a las personas dispuestas a realizar acciones a las cuales se les asocia un nivel de riesgo, en pro de un objetivo claramente definido (Davidsson y Honig, 2003).

Entrepreneur education (Educación para Emprendedores). Entendido como el proceso de capacitación a un determinado grupo poblacional, utilizando herramientas para crear entornos emprendedores, con el objetivo de desarrollar en ellos habilidades individuales que les permitan encaminarse en acciones emprendedoras (Peterman y Kennedy, 2003).

Entrepreneur student (Estudiante Emprendedor). Comprendido como el individuo que a través de la educación emprendedora adquiere la determinación y perseverancia, la habilidad para enfrentar obstáculos y una visión efectiva; que le permiten ejecutar acciones emprendedoras (Peterman y Kennedy, 2003).

Entrepreneur intention (Intenciones Emprendedoras). Corresponden a un término implementado inicialmente por Davidsson en 1995, con el cual se pretende establecer a través de variables económico-psicológicas la intención de un individuo para iniciar un negocio (Davidsson, 1995).

Los anteriores descriptores son combinados simultáneamente con un segundo grupo de descriptores (university, students, learning) los cuales complementan la correcta orientación de la búsqueda a través de la base de datos, focalizando los resultados de la misma de forma exclusiva en los temas de mayor interés para la presente investigación.

Finalmente, se define dentro de las prioridades de la búsqueda, que se debe excluir cualquier resultado que este orientado a estudios de medicina, por lo cual de define el descriptor medicine*, como una variable restrictiva en la ecuación propuesta.

De lo anterior resulta el planteamiento de la siguiente ecuación de búsqueda, la cual arrojó un total de 269 registros: 


\section{RESULTADOS Y DISCUSIÓN}

TITLE ("entrepreneur* education" W/2 ("entrepreneur* student*" W/2 university) OR (entrepreneur* W/2 learning* W/2 university) OR ("entrepreneur* intention" W/2 "university student*") AND KEY ("entrepreneur* education" W/2 university $W / 2$ students) OR ("entrepreneur* student*" W/2 university) OR (entrepreneur* W/2 learning* W/2 university) OR ("entrepreneur* intention" W/2 "university student*") AND NOT (medicine).

Posterior a esto se calculan los indicadores de cantidad, entre los que se encuentran: indicador de publicaciones anuales, cantidad de publicaciones acumuladas, publicación por revista, porcentaje acumulado de publicaciones por revista, cantidad de publicaciones de los primeros diez autores, porcentaje acumulado de las publicaciones de los primeros diez autores, ley de la productividad de autores, publicaciones por tipo de documento. Por otro lado, entre los indicadores de calidad, entre los que se encuentran: cantidad de citaciones por autor, cantidad de citaciones por revista y cantidad de citaciones por cada año.
Con base en los indicadores propuestos en la metodología, a continuación, se presentan los resultados para el presente análisis:

\section{Indicadores de cantidad}

En la primera sección, se presentan los indicadores bibliométricos de cantidad calculados para el tema de educación emprendedora, según la ecuación de búsqueda obtenida.

Los indicadores muestran un crecimiento en el interés por el tema a partir de 2006, a pesar de que en el siguiente año se presenta una disminución en la cantidad de artículos publicados sobre el tema, dicha disminución fue de 3 artículos, ya que se pasa de 8 a 5 publicaciones, por lo tanto, no se considera significativa. A partir de 2008, el interés por el tema de educación emprendedora se ha mantenido en constante incremento (ver Fig. 1).

Se evidencia que la cantidad de publicaciones acumuladas por año, presenta una curva de crecimiento exponencial, lo cual significa que la cantidad de publicaciones sobre educación emprendedora ha ido en aumento de forma significativa (ver Fig. 2).

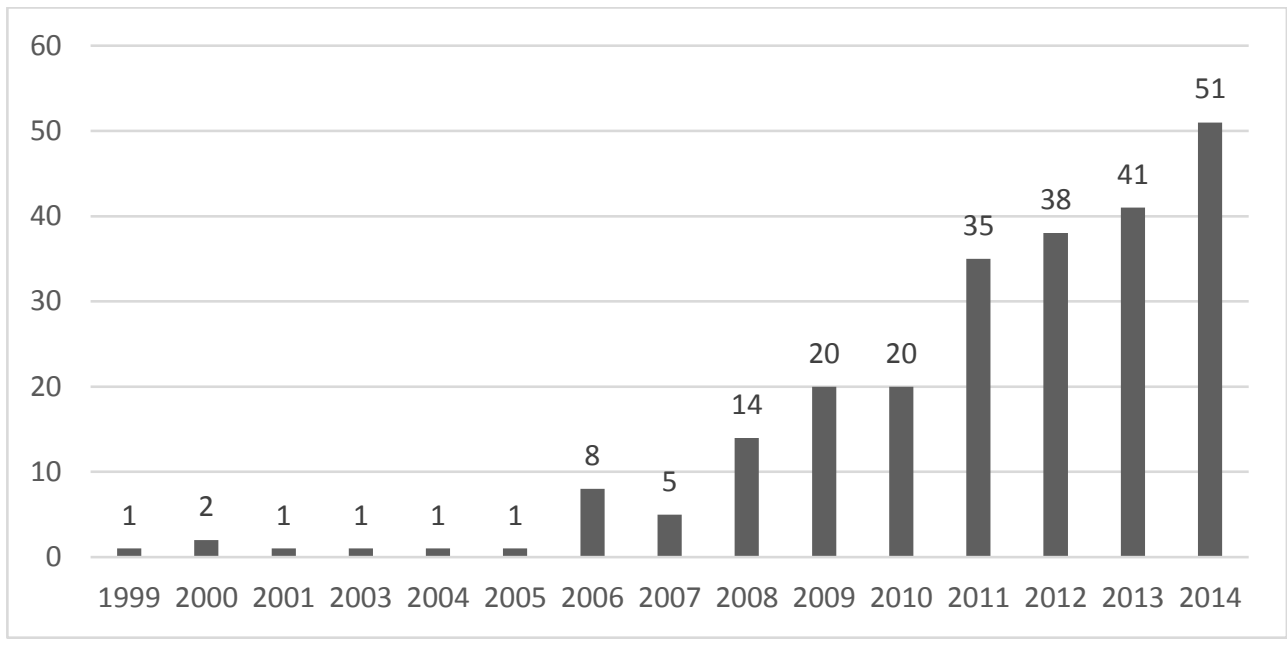

Figura 1. Cantidad de publicaciones por año

Figure 1. Number of publications per year

Fuente: elaboración propia. 


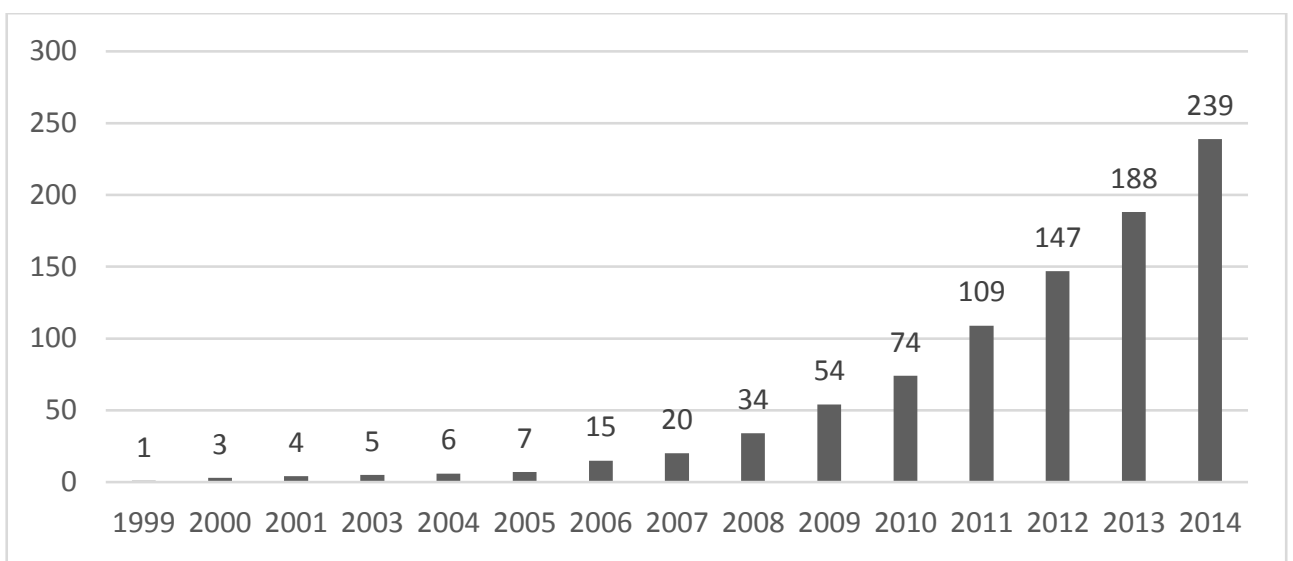

Figura 2. Cantidad de publicaciones acumuladas por año

Figure 2. Number of publications accumulated per year Fuente: elaboración propia.

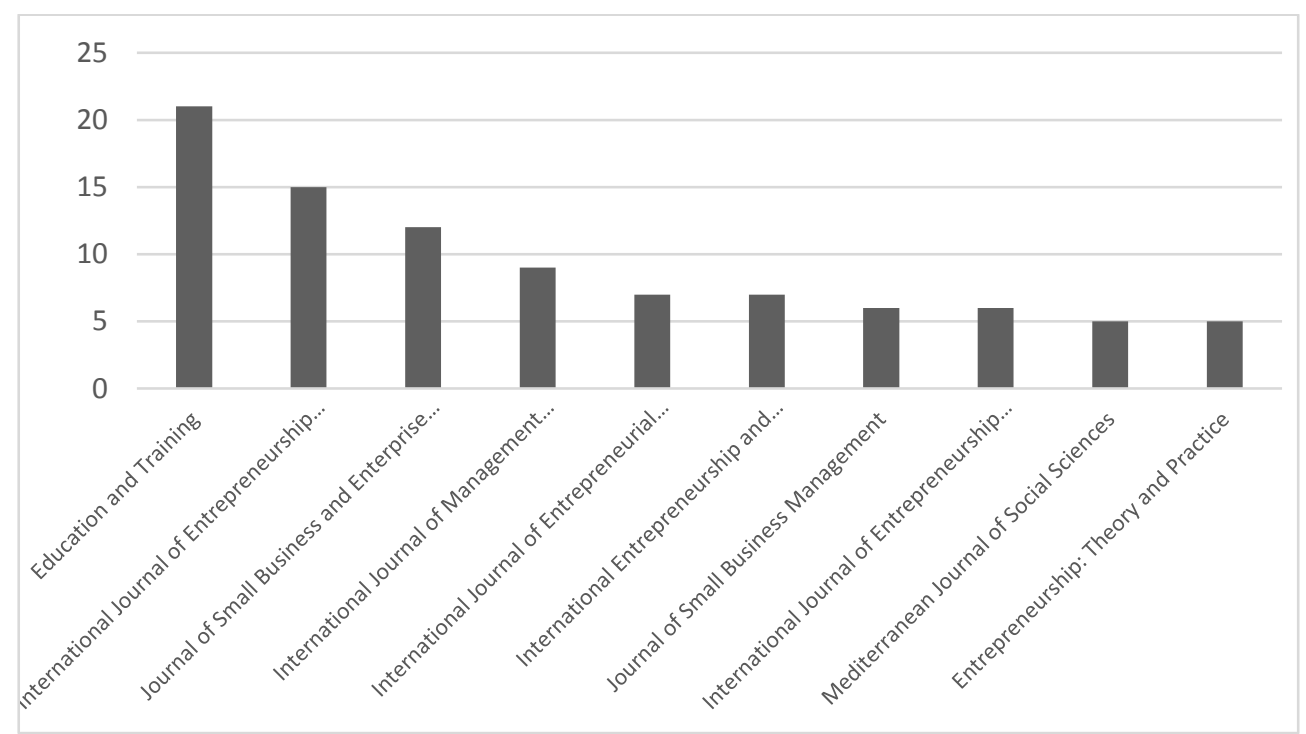

Figura 3. Cantidad de publicaciones por revista

Figure 3. Number of publications per journal

Fuente: elaboración propia.

Por otro lado, se pueden observar las diez revistas que más publicaciones tienen sobre el tema, donde la primera, Education and Training, cuenta con 21 publicaciones; seguida por International Journal of Entrepreneurship and Small Business, la cual cuenta con 15 publicaciones; la tercera revista en la línea, Journal of Small Business and Enterprise Development, la cual reporta 12 publicaciones (ver Fig. 3). Continuando con el análisis del porcentaje acumulado de publicaciones por revista (ver Fig. 4), se concluye que no existe Ley de Pareto, puesto que, el $64.67 \%$ de las revistas publica el $80 \%$ de los artículos, en vista de esto se procedió a separar el porcentaje de revistas por cuartiles, obteniendo que: el $3.33 \%$ de las revistas publica el $25 \%$ de los artículos, el 17.33 $\%$ de las revistas publica el $50 \%$ de los artículos, y finalmente las estadísticas arrojaron que el $55.33 \%$ de las revistas publica el $75 \%$ de los artículos. La información recogida deja ver que la distribución de artículos por revista es equitativa, dado que no predomina una sola revista para todos los registros exportados por la base de datos. 


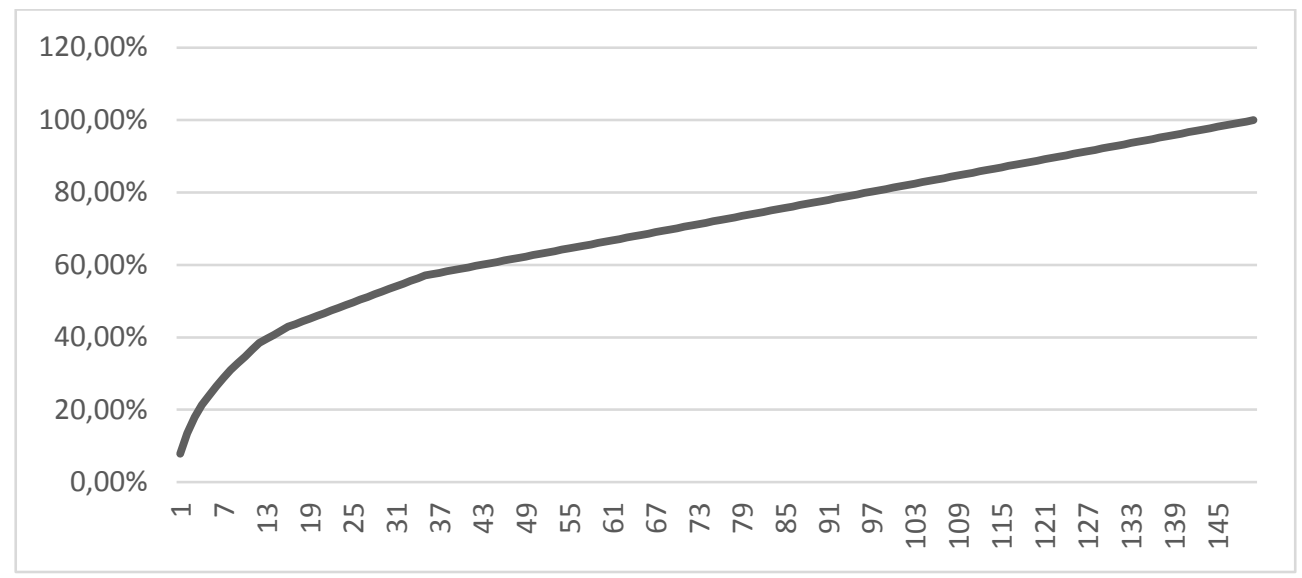

Figura 4. Porcentaje acumulado de publicaciones por revista

Figure 4. Cumulative percentage of publications by journal Fuente: elaboración propia.

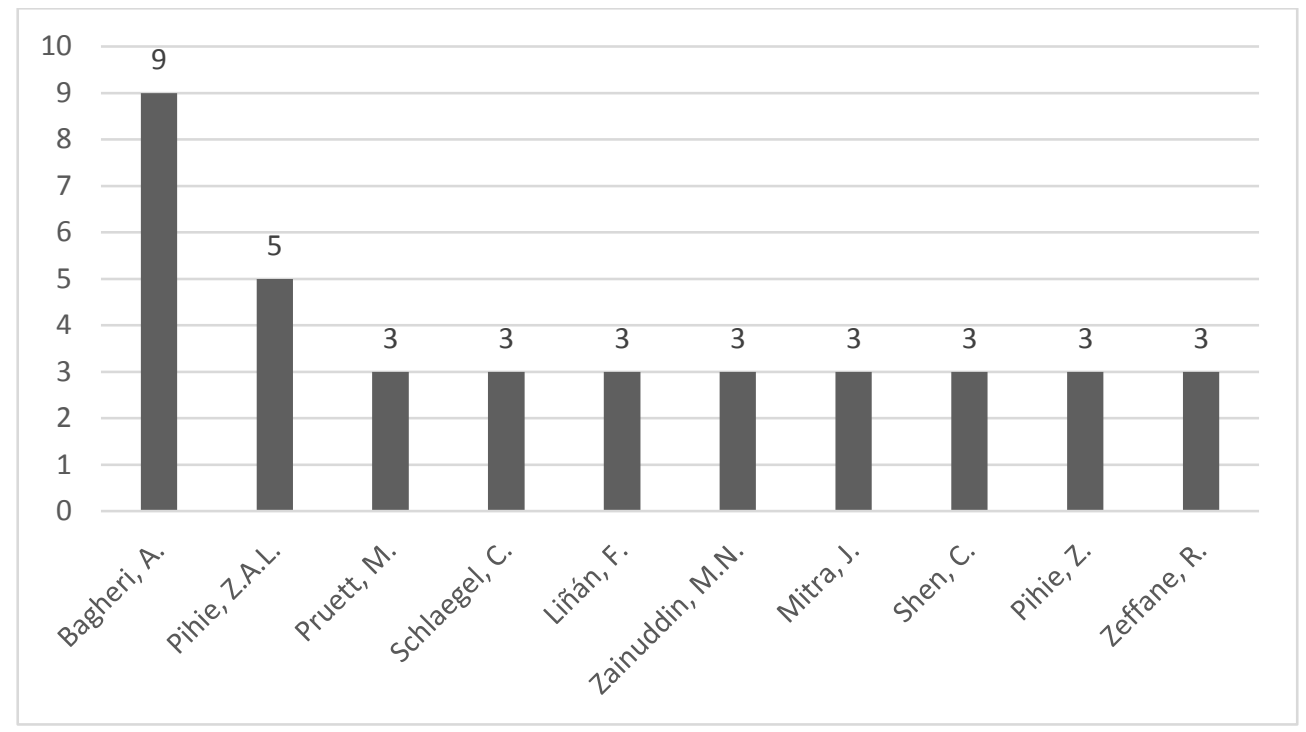

Figura 5. Cantidad de publicaciones de los primeros diez autores

Figure 5. Number of publications of the first ten authors

Fuente: elaboración propia.

Con respecto a los diez autores que más producciones tienen sobre el tema, quien encabeza la lista es el investigador Bagheri A., con 9 artículos publicados; seguido por Pihie Z., quien cuenta con 5 artículos; los 8 autores restantes cuentan con 3 publicaciones cada uno (ver Fig. 5).

En relación con lo anterior, la cantidad acumulada de publicaciones de los primeros diez autores (ver Fig. 6), muestra que no existe Ley de Pareto, puesto que el $76.43 \%$ de los autores publica el $80 \%$ de los artículos; dada esta situación, se procedió a separar el porcentaje acumulado de publicaciones para los primeros diez autores, obteniendo que: el $12.14 \%$ de los autores publica el $25 \%$ de los artículos, el 41.07 $\%$ de los autores publica el $50 \%$ de los artículos y finalmente el $70.71 \%$ de los autores publica el $75 \%$ de los artículos. La información recogida deja ver que la distribución de artículos por investigador tiene una distribución equitativa, evitando que predominen las publicaciones de un solo autor en la literatura. 
Como se puede observar en el Fig. 7, las publicaciones no están concentradas en pocas revistas. Posteriormente, se verificó si se cumplía la ley de productividad; la cual postula que a medida que aumenta el número de trabajos, el número de revistas disminuye. El coeficiente de determinación es mayor a 0.8, lo cual demuestra un buen ajuste al modelo planteado para la productividad.

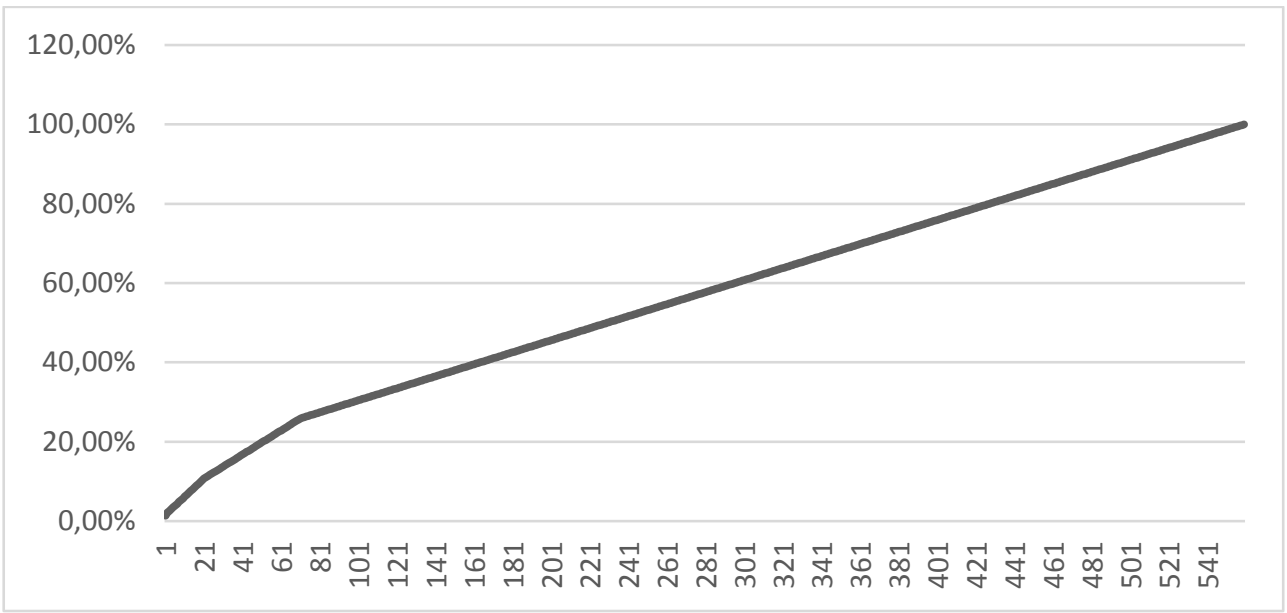

Figura 6. Porcentaje acumulado de las publicaciones de los primeros autores

Figure 6. Cumulative percentage of publications of the first authors Fuente: Elaboración propia.

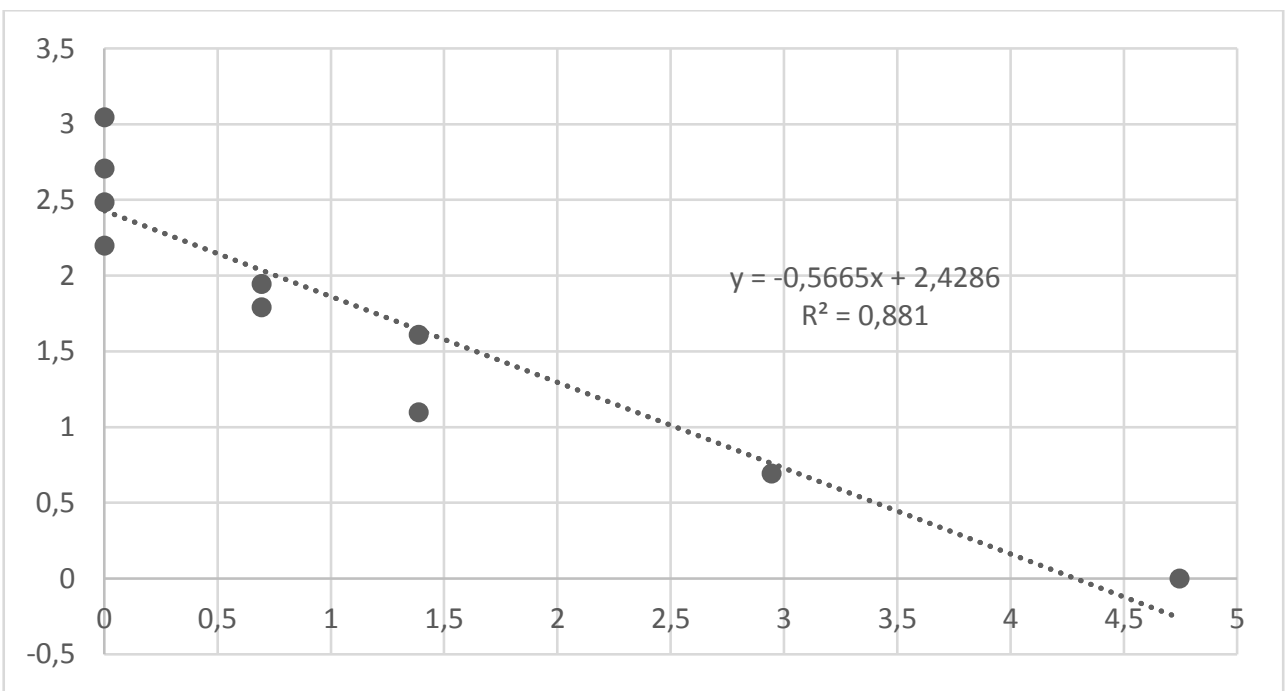

Figura 7. Ley de la Productividad para Revistas

Figure 7. Productivity Law for Journal

Fuente: elaboración propia. 
Esta misma lógica se aplica para validar la Ley de la Productividad de los autores (ver Fig. 8) la cual señala que el valor del coeficiente de regresión al cuadrado del ajuste del número acumulado de artículos, frente al logaritmo natural de los autores, es mayor a 0.9, lo que indica que se ajusta adecuadamente con el modelo de productividad.
Por último, cabe mencionar que el $78.36 \%$ de los registros obtenidos a partir de la ecuación de búsqueda corresponden a artículos y el $7.46 \%$ son artículos presentados en conferencias (conference papers); la participación de los demás tipos de documentos acerca de la educación emprendedora es del $16.42 \%$ (ver Fig. 9). Lo anterior puede ser un indicador de relevancia actual del tema, donde se evidencia que el principal medio de difusión del mismo son los artículos.

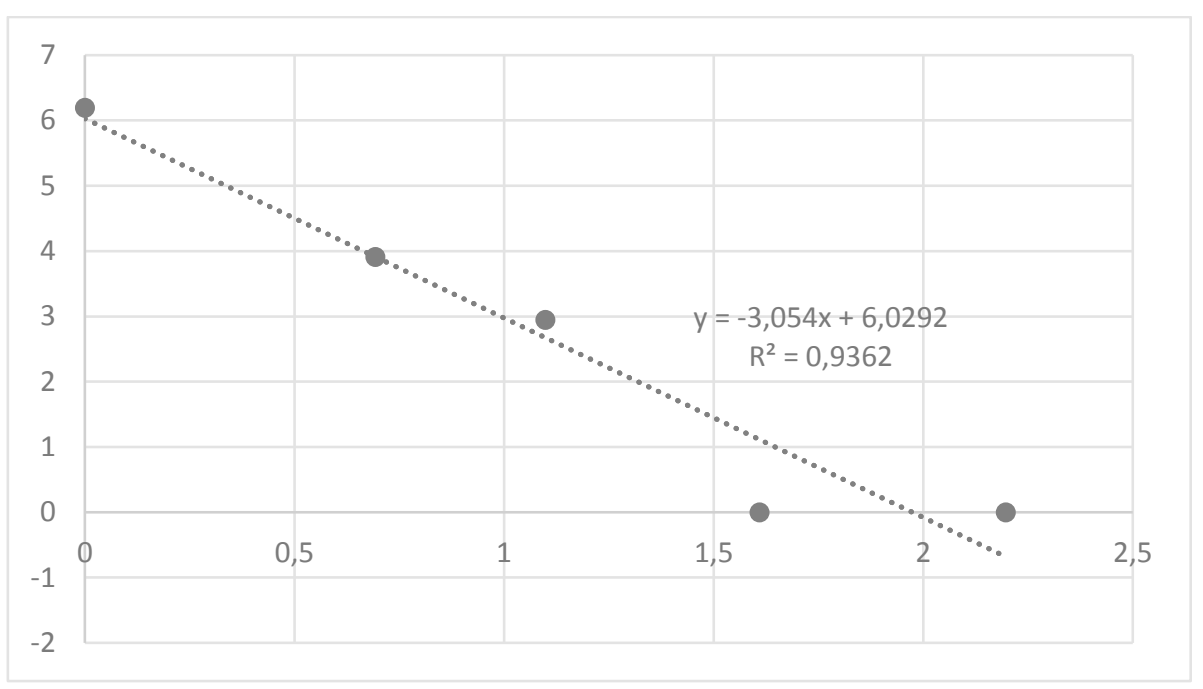

Figura 8. Ley de la productividad de autores

Figure 8. Authors productivity law Fuente: elaboración propia.

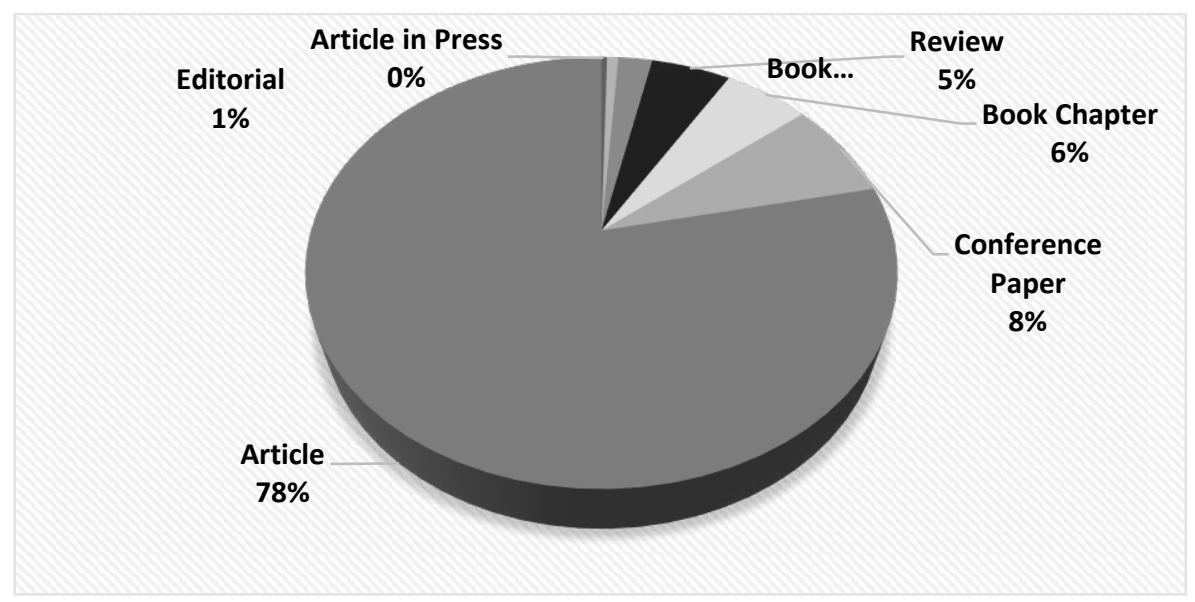

Figura 9. Publicaciones por tipo de documento

Figure 9. Publications by document type Fuente: elaboración propia. 


\section{Indicadores de calidad}

La segunda sección presenta los indicadores bibliométricos de cantidad calculados para el tema educación emprendedora, según la ecuación de búsqueda obtenida.

Con los indicadores de calidad es posible encontrar los diez autores con mayor cantidad de citaciones en el tema; en este sentido, Liñán F., es el que cuenta con la mayor cantidad de citaciones, con 163 (ver Fig. 10), seguido por Chen Y., con 150 publicaciones; el tercer investigador en la lista, Lichtenthaler $U$, registra 90 publicaciones. Los tres autores siguientes: Rich D. C., Robinson G. y Bednarz R., cuentan con 88 publicaciones respectivamente. Se observa, además, que solo el investigador Liñán F., aparece en la lista de los autores con mayor cantidad de publicaciones (ver Fig. 5).

Por otro lado, el indicador de impacto de las citaciones por revista (ver Fig. 11) presenta, como en el caso de los autores, una similitud frente al indicador de cantidad, ya que la revista Entrepreneurship: Theory and Practice, se encuentra en ambos indicadores (ver Fig. 3).

Finalmente, el análisis bibliométrico permite identificar que el tema no fue muy estudiado en sus dos primeros años de inicio, el año en el que generó mayor interés fue el 2001, donde se registra un total de 88 citaciones, sin presentar ninguna citación durante el siguiente año, 2003. En el lapso de 2004 a 2014, el año que presenta mayor número de citaciones es el 2007, con 25 citaciones (ver Fig. 12).

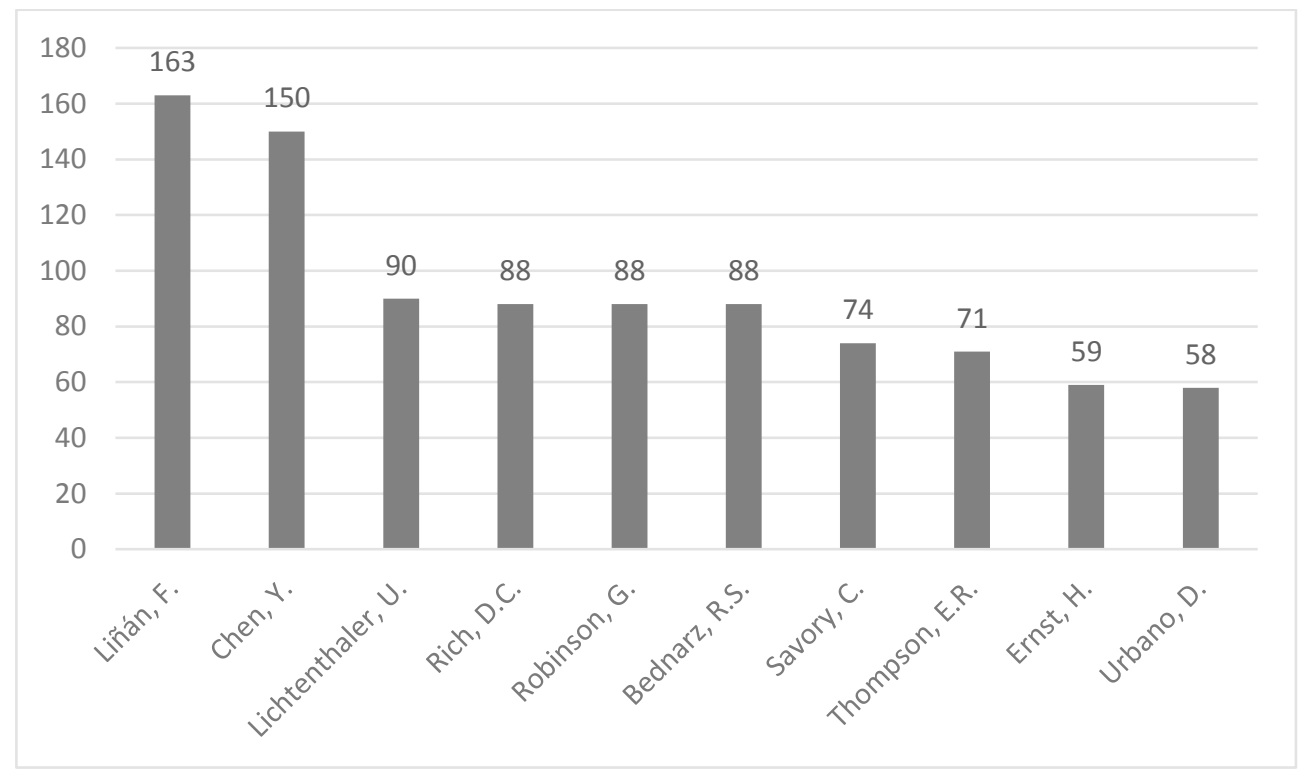

Figura 10. Cantidad de citaciones por autor

Figure 10. Number of citations per author

Fuente: elaboración propia. 


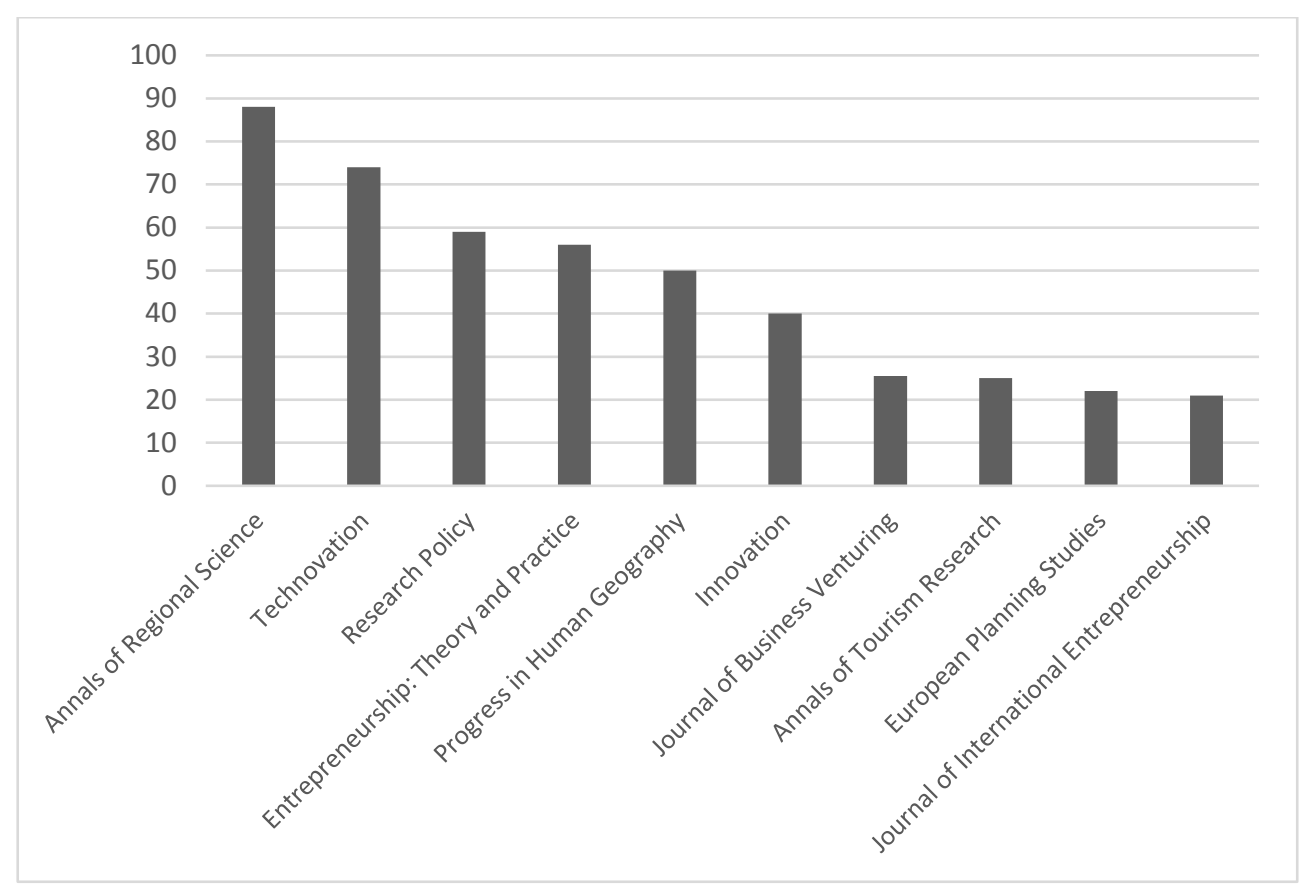

Figura 11. Cantidad de citaciones por revista

Figure 11. Number of citations per journal

Fuente: elaboración propia.

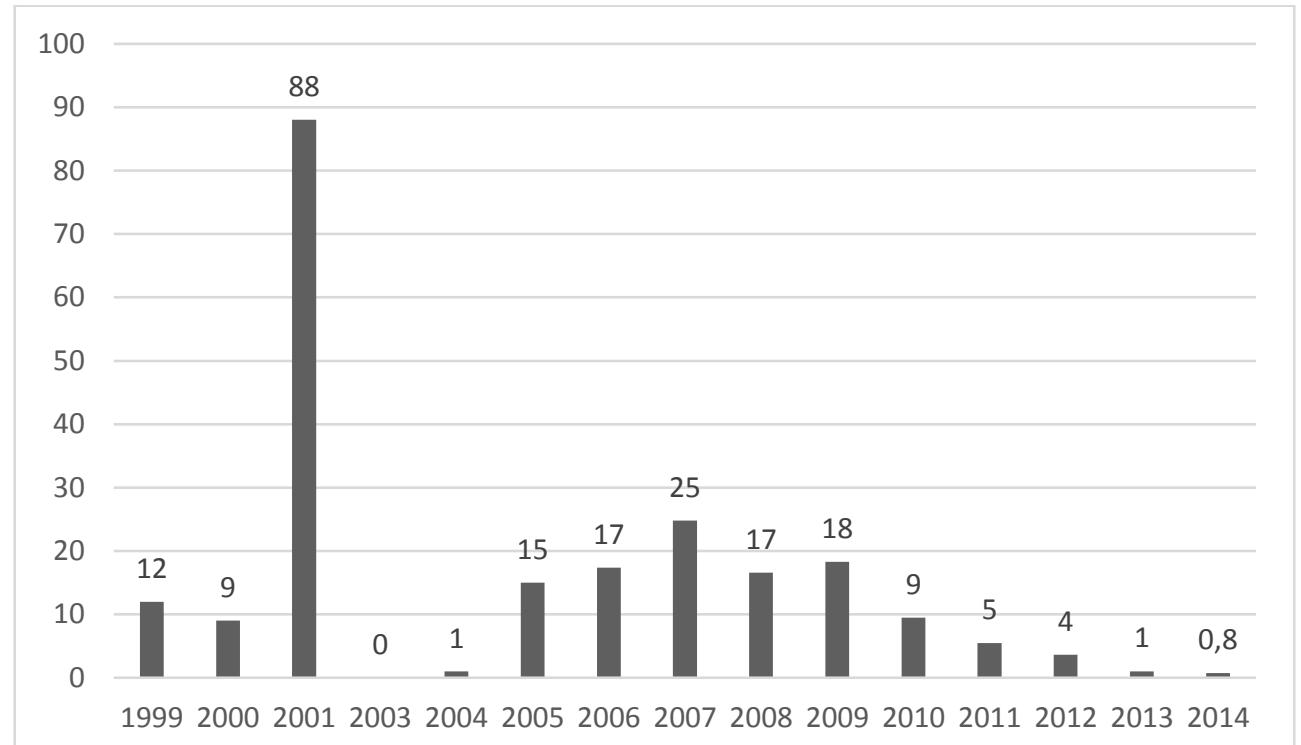

Figura 12. Cantidad de citaciones por cada año

Figure12. Number of citations for each year

Fuente: elaboración propia. 


\section{EVOLUCIÓN Y TENDENCIAS INVESTIGATIVAS}

A partir de los 269 registros que se obtuvieron con la ecuación de búsqueda, entre los años 1999 y 2014, se realizó un análisis de la evolución y las tendencias que han tenido los estudios realizados con respecto a la educación emprendedora en el contexto universitario. En el Figura 13 se puede observar dicha evolución de temáticas en torno al campo de estudio, asimismo, se pueden apreciar las tendencias que han surgido en relación con la educación empresarial en las universidades.
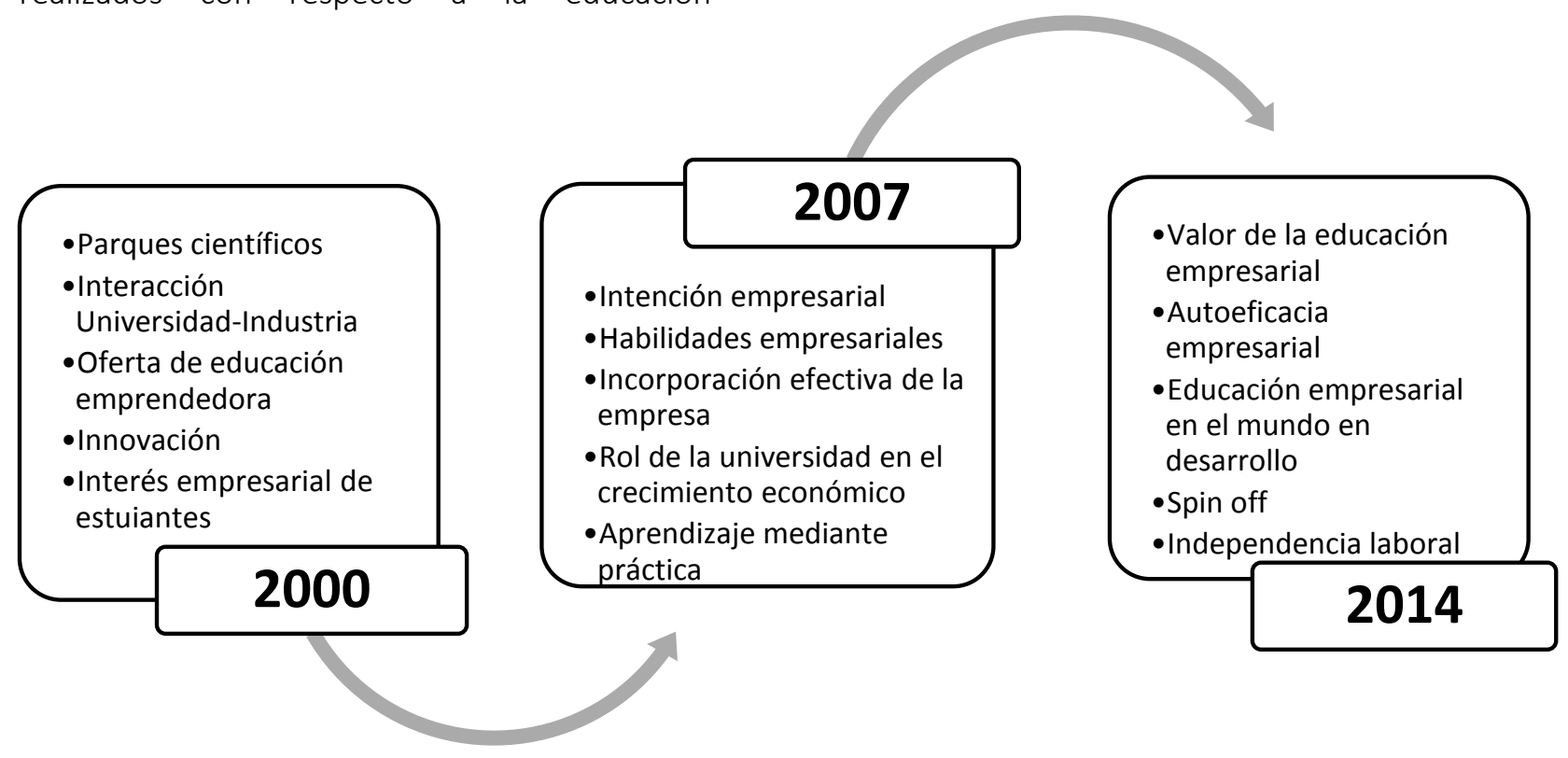

Figura 13. Cronología de la evolución del campo de estudio Figure 13. Chronology of the evolution of the field of study Fuente: elaboración propia.

En el Fig. 13 se puede observar que los estudios relacionados con la educación empresarial en el contexto universitario han abordado una diversidad de temáticas, mostrando la amplitud del campo bajo estudio y la variedad de perspectivas con las que puede ser estudiado. En sus inicios (año 2000), se observan temas de investigaciones orientados al vínculo de dos agentes sociales: la universidad (academia) e industria (empresas), cuya relación puede ser facilitada por los parques científicos (Vedovello, 2000), al ser espacios que promueven la creación y consolidación de empresas innovadas. Para este mismo tiempo, surgen temas investigativos que abordan la importancia de ofertar cursos de educación emprendedora, con el fin de satisfacer las necesidades empresariales de los estudiantes en cuanto a sus aspiraciones futuras
(Collins, Hannon y Smith, 2004). La innovación también es un tema central en los comienzos de los estudios de la temática, los cuales se enfocan principalmente en el aprendizaje de la innovación que se puede adquirir en las universidades para la creación de empresas (Mitra, 2000). Finalmente, se muestra interés por estudios que tratan sobre el interés de los estudiantes por construir su propio negocio.

En contraste, para el año 2007 se destacan estudios orientados a determinar los efectos que trae la implementación de la educación empresarial sobre las acciones emprendedoras. Por un lado, se estudia cómo este tipo de educación logra generar un impacto sobre la intencionalidad empresarial de los estudiantes (Pittaway y Cope, 2007) y al mismo tiempo ayuda 
a desarrollar habilidades empresariales. Por otra parte, se han llevado a cabo estudios sobre la incorporación efectiva de la empresa, lograda a través de los cursos sobre emprendimiento. Igualmente, para este periodo se destacan estudios sobre el rol de la universidad en el crecimiento económico de las regiones al brindar cursos orientados en el emprendimiento, mostrando gran relevancia en cursos prácticos que motiven hacia la acción emprendedora.

Finalmente, la evolución muestra que para el año 2014 se realizan investigaciones sobre el valor que tiene la educación empresarial, dado que, aunque muchos estudios han abordado temáticas en torno a la educación en emprendimiento, pocos lo hacen sobre el valor percibido de los estudiantes. Para este año, también se nota interés en temáticas como la autoeficacia empresarial (Shinnar, Hsu y Powell, 2014), educación empresarial en el mundo en desarrollo como medio que incentiva el crecimiento (Zhang, Duysters y Cloodt, 2014), spin off e independencia laboral.

\section{AGENDA DE INVESTIGACIÓN}

Con base en la evolución y tendencias investigativas representadas en el Figura 13, a continuación, se presenta una agenda de investigación con el objetivo de orientar el futuro de la investigación de la educación emprendedora en el contexto universitario.

- La autoeficacia empresarial es una variable que contribuye a la intención emprendedora, y que puede ser estimulada o fortalecida por la educación empresarial. Este concepto, que se refiere a la creencia de los individuos en cuanto a la capacidad que tienen para construir un negocio (Chen, Greene y Crick, 1998), ha generado en los últimos años gran interés y atención en las investigaciones por determinar el rol que tiene dicha variable dentro del proceso emprendedor.
- Dentro de los hallazgos, se resaltan los resultados que genera el emprendimiento en el contexto universitario. Uno de estos resultados son Isa "spin off", que consisten en la creación de nuevas empresas con conceptos tecnológicos y de investigación que han sido desarrollados dentro de una universidad (Hannibal, Evers y Servais, 2016). De esta manera, se ha resaltado la importancia del vínculo entre la academia y la industria, por lo que resulta importante determinar la construcción de esta relación y de qué manera pueden interactuar para contribuir al desarrollo económico de las regiones.

- Una de las razones para emprender consiste en solucionar problemas de empleabilidad, por lo que este concepto ha resultado valioso para fomentar la independencia de las personas en el ámbito laboral. En este sentido, ante las dificultades que viven actualmente los países en desarrollo, el papel de las universidades es apoyar y ayudar a los estudiantes a vincularse con el mundo laboral. Una de las formas en que las instituciones superiores pueden apoyar a los estudiantes es brindando educación emprendedora que les pueda abrir más oportunidades a los estudiantes en su vida profesional.

- Se ha cuestionado el valor y la eficacia de la educación emprendedora, por lo que ha surgido el interés de evaluar de qué forma se puede mejorar, con el fin de estimular a los estudiantes hacia la acción de emprender. Una de las maneras es realizar los cursos de forma más práctica y no realizar las clases de forma tradicional. En este sentido, aún quedan muchos vacíos que pueden ser cubiertos en futuras investigaciones en torno al tipo y metodología de educación emprendedora que se debe dar en las universidades. 


\section{CONCLUSIONES}

Frente a los resultados se observa que se presenta un notable interés por estudiar temáticas relacionadas con la educación emprendedora, reflejada en el crecimiento constante que han registrado las publicaciones en este campo de conocimiento lo que muestra el potencial que sigue planteando esta área de investigación y la necesidad de seguir incentivando la investigación en sub-temáticas emergentes como son: educación en innovación y gestión tecnológica, creación de spin-off e incubadoras de empresas de origen universitario.

Se observa que no solo las revistas enfocadas en emprendimiento han incentivado la publicación de artículos orientados a la educación emprendedora, este es el caso de revistas internacionales como Education and Training, International Journal of Entrepreneurial and Management Journal, Mediterranean Journal of Social Science. Por otro lado, las revistas enfocadas en el tema de emprendimiento que han buscado potencializar la publicación en esta temática se encuentran: International Journal of Entrepreneurship and Small Business, Journal of Small Business and Enterprise Development, International Journal of Entrepreneurial Behaviour and Research; sin embargo, la revista con mayor impacto en la temática (medida por el número de citaciones) es: Entrepreneurship Theory and Practice, la cual se encuentra entre las más importantes en el campo de emprendimiento, ubicándose entre los cuartiles más altos en el área de Business and Management, en Scopus.

Por otro lado, la información recogida deja ver que la distribución de artículos por autores es equitativa, es decir, no se identificó predominio o especialización de pocas revistas o autores en la temática, lo cual puede ser una muestra de que la temática aún está abierta a diferentes ópticas y teóricos que propongan nuevas perspectivas de contribución y análisis de la temática.
Es importante destacar que la mayor cantidad de publicaciones se ha difundido mediante artículos en revistas académicas arbitradas $(78,36 \%$ de los registros encontrados), lo que muestra que este medio de difusión ha tomado preponderancia frente a los papers de conferencias, libros y otro tipo de publicaciones. Este mecanismo de publicación tiene la ventaja de que ofrece la posibilidad de mayor visibilidad y divulgación como canal académico reconocido ya que las publicaciones se asocian a importantes bases académicas.

\section{REFERENCIAS}

Abdullah, A., y Abidin, Z. Z. (2014). Factors influencing graduates to start a business: $A$ case of Malaysian public institution of higher learning. En 23rd International Business Information Management Association Conference, IBIMA 2014. España.

Agudelo, D.; Bretón-López, J. y Buela-Casal, G. (2003). Análisis bibliométrico de las revistas de psicología clínica editadas en castellano. Psicothema, 15(4).

Arshad, M.; Farooq, O.; Sultana, N., y Farooq, M. (2016). Determinants of individuals' entrepreneurial intentions: a gendercomparative study. Career Development International, 21(4), 318-339.

Bienkowska, D., y Klofsten, M. (2012). Creating entrepreneurial networks: academic entrepreneurship, mobility and collaboration during $\mathrm{PhD}$ education. Higher Education, 64(2), 207-222.

Bouyssou, D., y Marchant, T. (2011). Bibliometric rankings of journals based on impact factors: An axiomatic approach. Journal of Informetrics, 5(1), 75-86.

Buela-Casal, G., Carretero-Dios, H., y SantosRoig, M. D. L. (2002). Estudio comparativo de 
las revistas de psicología en castellano con factor de impacto. Psicothema, 14(4).

Boeris, C. E. (2011). Las fuentes de datos en los estudios bibliométricos. En: /I Jornada de Intercambio y Reflexión acerca de la Investigación en Bibliotecología. Universidad Nacional de la Plata, Argentina.

Cadavid, L.; Awad, G., y Cardona, C.J. (2012). A bibliometric analysis of a modeled field for disseminating innovation. Estudios Gerenciales, 28(SPE), 213-236.

Cano, A., y Sánchez, A. (1998). Síntesis de estudios bibliométricos españoles en educación. Una dimensión evaluativa. Revista española de documentación científica, 21(3), 269-285.

Chamorro, E., Ceballos, H., y Hernández, R. (2012). Burton Clark y su concepción acerca de la universidad emprendedora. Tendencias: Revista de la Facultad de Ciencias Económicas y Administrativas, 13(2), 103-118.

Chen, C., Greene, P., y Crick, A. (1998). Does entrepreneurial self-efficacy distinguish entrepreneurs from managers? Journal of business venturing, 13(4), 295-316.

Clark, B. (2004). Delineating the character of the entrepreneurial university. Higher Education Policy, 17(4), 355-370.

Collins, L.; Hannon, P., y Smith, A. (2004). Enacting entrepreneurial intent: the gaps between student needs and higher education capability. Education+ training, 46(8/9), 454463.

Davidsson, P. (1995). Determinants of entrepreneurial intentions. En RENT XI Workshop. Piacenza, Italy.

Davidsson, P., y Honig, B. (2003). The role of social and human capital among nascent entrepreneurs. Journal of Business Venturing, 18(3), 301-331.

Entrialgo, M., e Iglesias, V. (2016). The moderating role of entrepreneurship education on the antecedents of entrepreneurial intention. International Entrepreneurship and Management Journal, 12(4), 1209-1232.

Etzkowitz, H., y Klofsten, M. (2005). The innovating region: toward a theory of knowledge-based regional development. R\&D Management, 35(3), 243-255.

Guerrero, M.; Urbano, D., Cunningham, J., y Organ, D. (2014). Entrepreneurial universities in two European regions: A case study comparison. The Journal of Technology Transfer, 39(3), 415-434.

Hall, C. (2011). Publish and perish? Bibliometric analysis, journal ranking and the assessment of research quality in tourism. Tourism Management, 32(1), 16-27.

Hamzah, H.; Yahya, Z.; Sarip, A., y Mohd Adnan, Y. (2016). Impact of entrepreneurship education programme (EEP) on entrepreneurial intention of real estate graduates. Pacific Rim Property Research Journal, 22(1), 17-29.

Hannibal, M.; Evers, N., y Servais, P. (2016). Opportunity recognition and international new venture creation in university spin-offsCases from Denmark and Ireland. Journal of International Entrepreneurship, 14(3), 345372.

Herman, E., y Stefanescu, D. (2017). Can higher education stimulate entrepreneurial intentions among engineering and business students? Educational Studies, 43(3), 312327. 
Horvath, Z. (2016). Assessing calling as a predictor of entrepreneurial interest. Society and Economy, 38(4), 513-535.

Jacob, M.; Lundqvist, M., y Hellsmark, H. (2003). Entrepreneurial transformations in the Swedish University system: the case of Chalmers University of Technology. Research Policy, 32(9), 1555-1568.

Kirkwood, J.; Dwyer, K., y Gray, B. (2014). Students' reflections on the value of an entrepreneurship education. The International Journal of Management Education, 12(3), 307-316.

Kyrgidou, L.; Mylonas, N., y Petridou, E. (2013). Identifying tomorrow's entrepreneurs: entrepreneurship education in Greece. World Review of Entrepreneurship, Management and Sustainable Development, 9(3), 352-364.

Kuratko, D. (2003). Entrepreneurship education: Emerging trends and challenges for the 21st century. White Paper, US Association of Small Business \& Entrepreneurship.

Kuratko, D. F.; Ireland, R. D.; Covin, J. G., y Hornsby, J. S. (2005). A Model of Middle-Level Managers' Entrepreneurial Behavior. Entrepreneurship Theory and Practice, 29(6), 699-716.

López-Cózar, E.; Torres-Salinas, D.; JiménezContreras, E., y Ruiz-Pérez, R. (2006). Análisis bibliométrico y de redes sociales aplicado a las tesis bibliométricas defendidas en España (1976-2002): temas, escuelas científicas y redes académicas. Revista española de documentación científica, 29(4), 493-524.

Mahieu, R. (2006). Agents of change and policies of scale: a policy study of entrepreneurship and enterprise in education (Doctoral dissertation, Svenska och samhällsvetenskapliga ämnen).
Maresch, D.; Harms, R.; Kailer, N., y WimmerWurm, B. (2016). The impact of entrepreneurship education on the entrepreneurial intention of students in science and engineering versus business studies university programs. Technological forecasting and social change, 104, 172-179.

Matsheke, O., y Dhurup, M. (2017). Entrepreneurial-related Programmes and Students' Intentions to Venture into New Business Creation: Finding Synergy of Constructs in a University of Technology. Science, Technology and Society, 22(2).

Mitra, J. (2000). Making connections: innovation and collective learning in small businesses. Education+ Training, 42(4/5), 228-237.

Monzurul, S.; Rafiul, S., y Rana, M. B. (2017). Entrepreneurship Education and University Students' Entrepreneurial Intentions in Bangladesh. Entrepreneurship: Concepts, Methodologies, Tools, and Applications, 221.

Naudé, W. (2013). Entrepreneurship and economic development. Research Paper, UNU-WIDER, United Nations University (UNU), No. 2008/20, ISBN 978-92-9230-0661.

Nieuwenhuizen, C., Groenewald, D., Davids, J., Rensburg, L. J., y Schachtebeck, C. (2016). Best practice in entrepreneurship education. Problems and Perspectives in Management, 3(2).

Panigrahi, A., y Joshi, V. (2015). Entrepreneurship Education and Economic Development: An Indian Perspective. En 11th Biennial Conference on Entrepreneurship.

Pittaway, L., y Cope, J. (2007). Entrepreneurship education: a systematic review of the evidence. International small business journal, 25(5), 479-510. 
Soria-Barreto, K., Zuniga-Jara, S., y Ruiz-Campo, S. (2016). Educación e Intención Emprendedora en Estudiantes Universitarios: Un Caso de Estudio. Formación universitaria, 9(1), 25-34.

Piñuel, M. C. (2014). UNX: A Latin American community of knowledge for entrepreneurship. INNOQUAL-International Journal for Innovation and Quality in Learning, 2(3).

Rasmussen, E. A., y Sørheim, R. (2006). Actionbased entrepreneurship education. Technovation, 26(2), 185-194.

Riese, H. (2011). Enacting entrepreneurship education: The interaction of personal and professional interests in mini-enterprises. Cambridge Journal of Education, 41(4), 445460.

Sanz-Valero, J., Veiga de Cabo, J., Rojo-Alonso, C., Wanden-Berghe, C., Espulgues Pellicer, J. X., Y Rodrigues Guilam, C. (2008). Los filtros metodológicos: aplicación a la búsqueda bibliográfica en la medicina del trabajo española. Medicina y seguridad del trabajo, 54(211), 75-83.

Saxenian, A. (1994). Regional advantage: Culture and competition in silicon valley and route 128. Cambridge, MA: Harvard University Press.

Shinnar, R. S., Hsu, D. K., y Powell, B. C. (2014). Self-efficacy, entrepreneurial intentions, and gender: Assessing the impact of entrepreneurship education longitudinally. The International Journal of Management Education, 12(3), 561-570.

Siddiqui, S., y Jan, S. (2017). Developing Entrepreneurial Intensity among Women Entrepreneurs of Jammu \& Kashmir: Model Building through Confirmatory Factor Analysis. Vision, 21(3), 295-304.
Sun, Q. (2017). Constructing Entrepreneurial Educational Ecosystem in Colleges and Universities Based on Culture of Innovation. AGRO FOOD INDUSTRY HI-TECH, 28(3), 850854.

Universidad de Granada (2017). Biblioteca electrónica - Scopus. Recuperado de: https://biblioteca.ugr.es/pages/biblioteca_el ectronica/bases_datos/scopus

Vedovello, C. (2000). Science parks and university-industry links: a comparative analysis between a British and a Portuguese experience. International journal of services technology and management, 1(4), 358-374.

Velasco, R. M. (2016). The making of an entrepreneur: aligning institutional paradigm to the industry needs. Journal of Business and Retail Management Research, 10(3).

Virgen Ortiz, V., Oliveros, C. E., y Betancourt Guerrero, B. (2014). Análisis bibliométrico del campo de formación de emprendedores. Cuadernos de Administración (Universidad del Valle), 30(52), 44-53.

Wang, C. K., y Wong, P. K. (2004). Entrepreneurial interest of university students in Singapore. Technovation, 24(2), 163-172.

Wiger, N. P., Chapman, D. W., Baxter, A., Y DeJaeghere, J. (2015). Context matters: A model of the factors associated with the effectiveness of youth entrepreneurship training. Prospects, 45(4), 533-547.

Wompner, F. (2008). Educación superior para el emprendimiento. Expansiva Chile, 1-16. 
Zhang, Y., Duysters, G., y Cloodt, M. (2014). The role of entrepreneurship education as a predictor of university students' entrepreneurial intention. International Entrepreneurship and Management Journal, 10(3), 623-641. 